\title{
Demonstration of the Multimaterial Coating Concept to Reduce Thermal Noise in Gravitational-Wave Detectors
}

\author{
Simon C. Tait $\odot,{ }^{1}$ Jessica Steinlechner $\odot,{ }^{1,2,3,4, *}$ Maya M. Kinley-Hanlon $\odot,{ }^{1}$ Peter G. Murray $\odot,{ }^{1}$ \\ Jim Hough $\odot,{ }^{1}$ Graeme McGhee $\odot,{ }^{1}$ Felix Pein, ${ }^{2}$ Sheila Rowan, ${ }^{1}$ Roman Schnabel $\odot,{ }^{2}$ Cassady Smith $\odot,{ }^{1}$ \\ Lukas Terkowski®, ${ }^{2}$ and Iain W. Martin ${ }^{1}$ \\ ${ }^{1}$ SUPA, School of Physics and Astronomy, University of Glasgow, Glasgow G12 8QQ, Scotland \\ ${ }^{2}$ Institut für Laserphysik und Zentrum für Optische Quantentechnologien, Universität Hamburg, \\ Luruper Chaussee 149, 22761 Hamburg, Germany \\ ${ }^{3}$ Maastricht University, P.O. Box 616, 6200 MD Maastricht, Netherlands \\ ${ }^{4}$ Nikhef, Science Park 105, 1098 XG Amsterdam, Netherlands
}

(Received 11 January 2020; revised 10 April 2020; accepted 20 May 2020; published 1 July 2020)

\begin{abstract}
Thermal noise associated with the mechanical loss of current highly reflective mirror coatings is a critical limit to the sensitivity of gravitational-wave detectors. Several alternative coating materials show potential for reducing thermal noise, but cannot be used due to their high optical absorption. Multimaterial coatings have been proposed to enable the use of such materials to reduce thermal noise while minimizing their impact on the total absorption of the mirror coating. Here we present experimental verification of the multimaterial concept, by integrating aSi into a highly reflective $\mathrm{SiO}_{2}$ and $\mathrm{Ta}_{2} \mathrm{O}_{5}$ multilayer coating. We show a significant thermal noise improvement and demonstrate consistent optical and mechanical performance. The multimaterial coating survives the heat treatment required to minimize the absorption of the aSi layers, with no adverse effects from the different thermomechanical properties of the three materials.
\end{abstract}

DOI: 10.1103/PhysRevLett.125.011102

Introduction.-A number of gravitational-wave signals from binary black-hole mergers [1-5] and from a neutronstar merger [6] have been detected during the first two observing runs of the Advanced LIGO [7] and Advanced Virgo [8] gravitational-wave detectors. At their most sensitive frequencies, these detectors are limited by thermal noise arising from the amorphous highly reflective (HR) multilayer coatings applied to the interferometer mirrors. Several more-sensitive detectors are planned $[9,10]$, aiming to establish black hole populations, potentially uncover deviations from general relativity, clarify the expansion rate of the Universe and investigate the nature of black holes and other exotic sources [9].

All future detectors will require improved optical coatings to enable current coating thermal noise limits to be surpassed. The critical coating properties are the optical absorption, and the mechanical loss to which the thermal noise power spectral density is proportional. The coatings currently used in Advanced LIGO and Advanced Virgo are made from silica $\left(\mathrm{SiO}_{2}\right)$ and tantala doped with titania $\left(\mathrm{TiO}_{2}: \mathrm{Ta}_{2} \mathrm{O}_{5}\right)[7,8,11-14]$. Many approaches to develop coatings with reduced thermal noise and low optical absorption at the ppm level are being investigated, such as understanding correlations between atomic structure and material properties [15-19], elevated temperature deposition [20], different dopants and doping concentrations $[13,21]$, nanolayer structures $[22,23]$, crystalline coatings [24], alternative amorphous materials [21,25,26], and optimizing the layer thicknesses [27].
Amorphous silicon (aSi) is one of the most promising options to replace $\mathrm{Ta}_{2} \mathrm{O}_{5}$ in HR coatings. It can have very low mechanical loss [28] and the high refractive index enables significantly thinner coatings to be made, which further reduces the thermal noise. However, aSi shows too high optical absorption. Recent work has shown that the absorption can be reduced by heat treatment [29], by using a wavelength of $2 \mu \mathrm{m}$ [30] and by optimizing the deposition procedure [31], but it is still not low enough to meet the requirements of gravitational-wave detectors.

Another option that has been proposed to allow the use of aSi, even with current absorption levels, is a "multimaterial" coating design $[32,33]$. In such a design, some low-absorption layers are used at the top of the coating stack, to reflect the majority of the incident laser power. Higher absorbing materials are used in the lower parts of the stack where there is little laser power left to be absorbed. This allows the replacement of some relatively high-loss $\mathrm{Ta}_{2} \mathrm{O}_{5}$ layers with low-loss aSi layers, reducing the total mechanical loss (and thus thermal noise) of the coating stack. Several multimaterial coating designs have been proposed [25,26,31,34], but not experimentally demonstrated.

In practice, multilayer coatings are complex physical systems - which get more complicated the more different materials are involved. In particular, the differing thermalexpansion coefficients of each material make it interesting to investigate whether heat treatment damages the coating. Furthermore, there is evidence that material properties such 
as mechanical loss can be altered by stress [35]. The optical absorption might be affected by interface effects. Therefore, an experimental test is required to verify the theoretical prediction of how the various layers interact.

In this Letter, we experimentally prove the multimaterial coating concept by presenting mechanical loss and optical absorption measurements made on a prototype multimaterial coating. This coating is made of 5 bilayers of $\mathrm{SiO}_{2}$ and $\mathrm{Ta}_{2} \mathrm{O}_{5}$ followed by 5 more bilayers in which the $\mathrm{Ta}_{2} \mathrm{O}_{5}$ is replaced by aSi; see Fig. 1. Throughout this Letter we refer to the 5 double layers of $\mathrm{SiO}_{2} / \mathrm{Ta}_{2} \mathrm{O}_{5}$ as upper stack, to the 5 double layers of $\mathrm{SiO}_{2} / \mathrm{aSi}$ as lower stack and to all 10 double layers as full stack.

By comparing to our upper stack, we show that the incorporation of aSi layers reduces thermal noise of the full multimaterial stack by a factor of 2 compared to a pure $\mathrm{SiO}_{2} / \mathrm{Ta}_{2} \mathrm{O}_{5}$ coating with approximately the same reflectivity (deposited using the same technique). Because of the reflectivity of the upper stack, the absorption in the full multimaterial coating is more than a factor 20 below that of a pure $\mathrm{SiO}_{2} / \mathrm{aSi}$ coating. The absorption behaves similarly with heat treatment to the absorption observed in an $\mathrm{aSi} / \mathrm{SiO}_{2}$ bilayer [29]. Heat treatment up to $600^{\circ} \mathrm{C}$, which is above the temperature at which the absorption of $\mathrm{aSi}$ minimizes, is possible without causing damage (e.g., cracks) to the coating.

Coating transmission.-The coatings studied here were deposited using an ion plating technique [36] that can produce aSi with low optical absorption at $2 \mu \mathrm{m}$ and low mechanical loss [30]. Disks of diameter $76.2 \mathrm{~mm}$ and thickness $2.54 \mathrm{~mm}$ were used for the mechanical loss measurements [37]. For the absorption measurement, samples of diameter 25.4 and thickness $6.35 \mathrm{~mm}$ were used. The majority of absorption samples were made of Corning 7980 silica. Corning 7980 has significant absorption at $2 \mu \mathrm{m}$, making it an unsuitable substrate for absorption measurements of our low-absorption upper stack. Therefore a few Corning 7979 samples, which have lower absorption at $2 \mu \mathrm{m}$ due to lower $\mathrm{OH}$ content, were used.

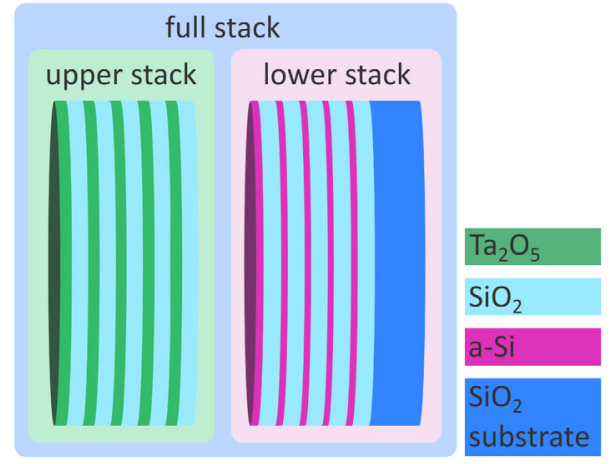

FIG. 1. Schematic of the multimaterial coating: The full stack is composed of the upper stack $\left(\mathrm{SiO}_{2}\right.$ and $\left.\mathrm{Ta}_{2} \mathrm{O}_{5}\right)$ and the lower stack (aSi and $\mathrm{SiO}_{2}$ ).
The lower stack was deposited onto a large set of samples. After deposition, some samples were removed from the coating chamber (providing the lower stack sample set), and replaced with new, uncoated samples. The upper stack was then deposited. This resulted in a set of samples coated with each of the three stacks, and ensured that the two components of the full-stack coating were identical to the separate upper and lower stacks.

The full stack was designed to provide a transmission of $<10 \mathrm{ppm}$, similar to end test masses used in gravitationalwave detectors. Figure 2 shows measured spectra of the three coatings in an as-deposited state. While the transmission $T$ was measured, the graph shows $1-T$, which is equivalent to the sum of reflectivity $R$ and absorption $A$, with $R+T+A=1$. On the scale used in this plot, $A$ is negligibly small.

The pink line in Fig. 2 shows $1-T$ for the lower stack. Because of the high refractive index of aSi, this coating shows a broad, highly reflective plateau. The green line shows $1-T$ for the upper stack. Because of the lower refractive index of $\mathrm{Ta}_{2} \mathrm{O}_{5}$, the reflectivity of this coating is significantly lower than that of the lower stack, with a narrower plateau. The blue line shows $1-T$ of the full stack.

Optical absorption.-To reduce their optical absorption, HR coatings often get heat treated following deposition [38]. Previous work indicates that the absorption of aSi is particularly strongly dependent on heat treatment, and can be reduced by a factor of up to 50 by heating to a temperature between $400{ }^{\circ} \mathrm{C}$ and $500^{\circ} \mathrm{C}$ [29-31]. The exact temperature at which the minimum absorption occurs, and the improvement factor, vary slightly depending on the coating deposition procedure used.

To investigate the effect of heat treatment on the multimaterial coating, we heat treated samples coated with the full stack and the lower stack at $100^{\circ} \mathrm{C}, 200^{\circ} \mathrm{C}, 300^{\circ} \mathrm{C}$, $400^{\circ} \mathrm{C}, 500^{\circ} \mathrm{C}$, and $600{ }^{\circ} \mathrm{C}$. For consistency checks, the absorption of the upper stack was also measured for the asdeposited coating and after heat treatment at $500{ }^{\circ} \mathrm{C}$ [40]. Heat treatment was carried out for $3 \mathrm{~h}$ in air. Coatings can

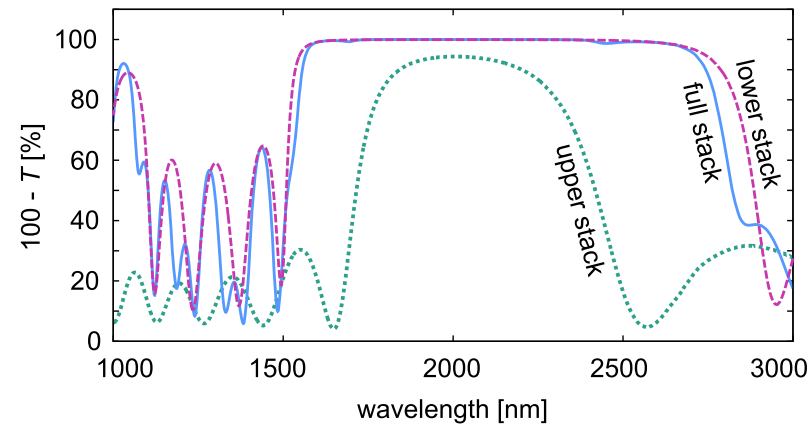

FIG. 2. Spectra of the three coatings: the $y$ axis shows $100 \%$ minus the measured transmission $T$. This is equivalent to the sum of the reflection $R$ and the absorption $A$-which can be assumed to be small on this scale and in this wavelength range. 
sometimes exhibit damage such as cracking or delamination, particularly following heat treatment. Microscope images of all samples were taken, and no evidence for either cracking or delamination was observed.

The optical absorption of the three stacks was measured at $2 \mu \mathrm{m}$ using photothermal common-path interferometry [41], which is an optical technique for measuring the thermal effect of absorbed laser power.

Figure 3 shows the measured absorption as a function of heat-treatment temperature for the lower stack (pink squares), the upper stack (green diamonds), and the full stack (blue circles). None of the coating stacks shows a significant change in absorption at heat-treatment temperatures of $\leq 200^{\circ} \mathrm{C}$. We believe that this is because the substrates reach a temperature of $\approx 200{ }^{\circ} \mathrm{C}$ during deposition, and therefore no changes occur with further heat treatment at or below this temperature. For heat treatment above $200{ }^{\circ} \mathrm{C}$, the absorption of the lower stack reduces by a factor of $\approx 7$ to a minimum value of $181 \mathrm{ppm}$ at $500^{\circ} \mathrm{C}$. The upper stack reduces from 4.8 to $3.0 \mathrm{ppm}$. The full stack also reduces in absorption, by a factor of $\approx 5$ to a minimum value of $8.1 \mathrm{ppm}$ at $500^{\circ} \mathrm{C}$, which is a factor of 22 lower than the absorption of the lower stack.

To allow comparison of our measurements with other coatings, it is necessary to calculate the extinction coefficient $k$ of the coatings. $k$, to which the absorption is proportional, is the imaginary part of the refractive index. For aSi, $k$ was estimated from the absorption of the lower stack under the assumption that the absorption of $\mathrm{SiO}_{2}$ is negligible compared to aSi. Similarly, $k$ of $\mathrm{Ta}_{2} \mathrm{O}_{5}$ was estimated from the absorption of the upper stack. The light field in the full stack was simulated using these $k$ values to predict the absorption of the full stack from the two partial stacks. This prediction is shown by the red circles in Fig. 3 for the coatings asdeposited and heat treated at $500^{\circ} \mathrm{C}\left(k \approx 3.4 \times 10^{-4}\right.$ for aSi and $k \approx 1.2 \times 10^{-6}$ for $\mathrm{Ta}_{2} \mathrm{O}_{5}$ ), and agrees closely with the measured absorption of the full stack.

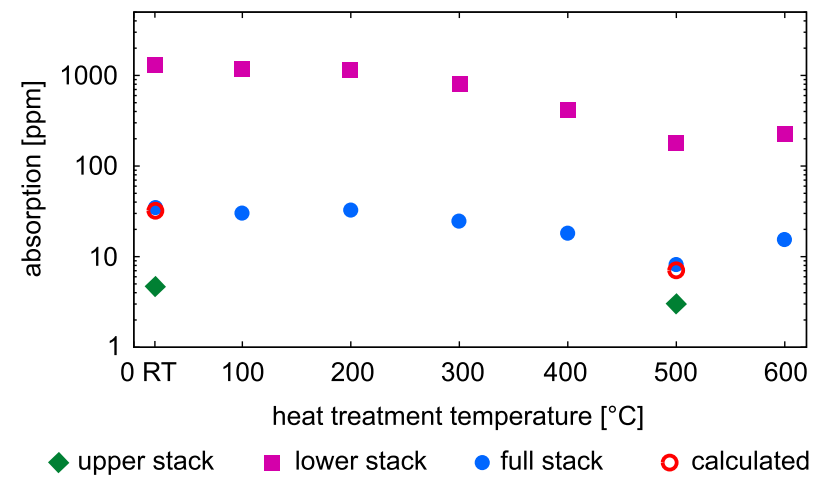

FIG. 3. Measured absorption of the three coatings as a function of heat-treatment temperature. Also shown is the predicted absorption of the full stack, based on the measurements of the upper and lower stacks. (Note that the samples reach a temperature of $\approx 200{ }^{\circ} \mathrm{C}$ during deposition.)
Mechanical loss.—Fused silica disks coated with each of the three stacks were mounted on a nodal support [42], in which the disk is balanced on a silicon spherical cap of radius of curvature $60.44 \mathrm{~mm}$. The mechanical loss of the three coating stacks was measured using a ring-down technique, in which the free-amplitude decay of vibrational modes of the disks is measured. For vibrational modes with a node at the center of the disk, this is a highly effective method of minimizing external frictional damping and ensuring that only losses due to internal friction in the disk are measured. The mechanical loss of approximately eight modes of each disk, between $\approx 2.8$ and $\approx 30 \mathrm{kHz}$, was measured before and after deposition of the coatings. To obtain the coating loss, the difference in coated and uncoated loss was scaled by the ratio of the elastic energy stored in the substrate to that stored in the coating [43]. This ratio was calculated using finite element analysis (COMSOL). Figure 4 shows a photograph of a disk coated with the full stack in the setup used for loss measurements and of a COMSOL model of an excited mode of the disk.

The loss was found to have some variation with frequency, which is likely to partially originate from a different split into bulk and shear motion dependent on the mode shape [44]. Figure 5 shows the average coating loss, calculated over all measured modes, for each stack as a function of heat treatment-see Table I for the results of the coating as deposited and after heat treatment at $500^{\circ} \mathrm{C}$. As expected, the lower stack has the lowest loss, due to the low loss of the $\mathrm{SiO}_{2}$ and aSi layers. The loss of the upper stack is significantly higher, due to the high loss of the $\mathrm{Ta}_{2} \mathrm{O}_{5}$ layers.

The loss of all three stacks decreased with heat treatmentby $17 \%$ for the upper stack, by $36 \%$ for the lower stack, and by $26 \%$ for the full stack. This trend is as predicted from previous measurements of the individual materials $[20,30,49]$. Also shown in Fig. 5 is the loss of the full stack predicted from the measurements of the upper and lower stacks (red circles). The prediction shows excellent agreement with the measurements (blue dots). The average loss of the full stack, at the optimum heat-treatment temperature, is

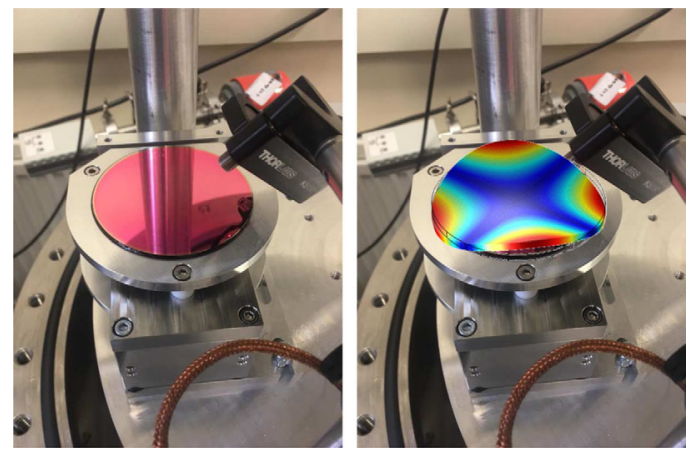

FIG. 4. Photo of the full stack multimaterial coating in the setup for mechanical loss measurements (left) and a COMSOL simulation of the disk with an excited butterfly mode at $2.7 \mathrm{kHz}$ (right). 


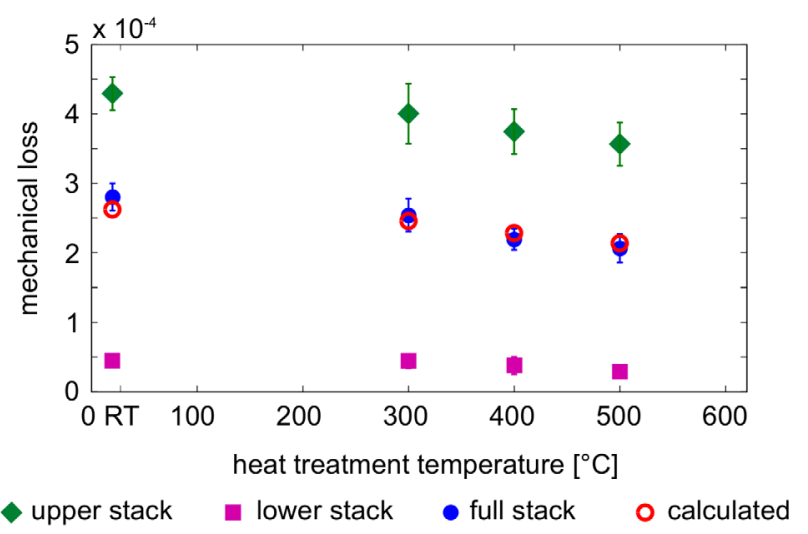

FIG. 5. Measured mechanical loss of the three stacks as a function of heat-treatment temperature. Also shown is the predicted loss of the full stack calculated from the measurements of the upper and lower stacks. (Note that the samples reach a temperature of $\geq 200^{\circ} \mathrm{C}$ during deposition.)

$\approx 42 \%$ lower than the loss of the upper stack, demonstrating the value of including aSi in the coating design [50].

Coating thermal noise.-In addition to loss, the thickness of a mirror coating is a crucial parameter in determining the thermal noise, and the high index of aSi brings significant benefits in enabling a thinner coating to achieve the same reflectivity. Thermal noise also depends on the Young's moduli of the coating materials. In this section, we present thermal noise calculations to compare the overall performance of our coatings.

Coating thermal noise amplitude spectral density is given by [33]

$$
x(f)=\sqrt{\frac{2 k_{B} T}{\pi^{2} f} \frac{1}{w^{2}} \frac{1-\sigma_{\mathrm{sub}}-2 \sigma_{\mathrm{sub}}^{2}}{Y_{\mathrm{sub}}} \sum_{j} b_{j} d_{j} \phi_{j}},
$$

where $k_{B}$ is the Boltzmann constant, $T$ the mirror temperature, $f$ the frequency, and $w$ the radius of the interferometer laser beam on the coating. $Y_{\text {sub }}$ and $\sigma_{\text {sub }}$ are the Young's modulus and the Poisson ratio of the substrate. $d$ is the coating thickness and $\phi$ the coating mechanical loss. The index $j$ refers to the material parameters of the $j$ th layer in the coating (starting from the outermost layer). For the calculations, we assume here that the mechanical losses associated with bulk motion and shear motion [44] are approximately equal $\left(\phi_{\text {bulk }} \approx \phi_{\text {shear }} \approx \phi\right) . b_{j}$ (The first factor in square brackets is a correction [51] to the expression for $b_{j}$ given by Yam, with which the authors of the Yam paper agree) is a weighting factor described by

$$
\begin{aligned}
b_{j}= & {\left[\frac{\left(1-2 \sigma_{j}\right)\left(1+\sigma_{j}\right)}{\left(1-2 \sigma_{s}\right)\left(1+\sigma_{s}\right)}\right] \frac{1}{1-\sigma_{j}} } \\
& \times\left[\left(1-n_{j} \frac{\partial \theta_{c}}{\partial \theta_{j}}\right)^{2} \frac{Y_{s}}{Y_{j}}+\frac{\left(1-\sigma_{s}-2 \sigma_{s}^{2}\right)^{2}}{\left(1+\sigma_{j}\right)^{2}\left(1-2 \sigma_{j}\right)} \frac{Y_{j}}{Y_{s}}\right],
\end{aligned}
$$

where $\sigma_{j}$ and $\sigma_{s}$ are the Poisson's ratios of each coating layer and the substrate. $n_{j}$ is the refractive index of the $j$ th layer and $\partial \theta_{\text {coat }} / \partial \theta_{j}$ describes the sensitivity of the total coating phase $\theta_{\text {coat }}$ to fluctuations in the round-trip phase $\theta_{j}$ in each layer.

$x(f)$ was calculated for 19 bilayers of $\mathrm{SiO}_{2} / \mathrm{Ta}_{2} \mathrm{O}_{5}$ which provide a similar reflectivity to our full multimaterial stack $(\approx 99.999 \%)$. We used the average Young's modulus of the two materials and the loss measured on our upper stack after heat treatment at $500{ }^{\circ} \mathrm{C}$ (see Table I).

Layers contribute more to coating thermal noise the lower in the stack they are located, as discussed by Ref. [44] in the derivation of Eq. (2). This is relevant here due to the lower loss of the lower stack. The coating thermal noise of the multimaterial coating reduces by (49.4 \pm 0.5$) \%$ compared to the pure $\mathrm{SiO}_{2} / \mathrm{Ta}_{2} \mathrm{O}_{5}$ coating with similar reflectivity.

Summary.-We have measured the optical absorption and the mechanical loss of a multimaterial coating consisting of an upper stack of $\mathrm{SiO}_{2} / \mathrm{Ta}_{2} \mathrm{O}_{5}$ and a lower stack of $\mathrm{SiO}_{2} / \mathrm{aSi}$. We showed that the results for the partial stacks and the full stack are self-consistent. Furthermore, we observed no damage evidence of cracking or delamination

TABLE I. Measured losses for our three stacks and material parameters used for calculating coating thermal noise. For the individual materials, the thickness $t$ of one quarter-wave layer is given. The effective Young's modulus $Y$ and Poisson ratio $\sigma$ of multilayer coatings was obtained using a thickness-weighted average of the values for each component layer [45].

\begin{tabular}{lcccccc}
\hline \hline & & & & & \multicolumn{2}{c}{$\phi \times 10^{-4}$} \\
\cline { 4 - 7 } & $\mathrm{Y}(\mathrm{GPa})$ & $d(\mathrm{~nm})$ & $n$ at $2000 \mathrm{~nm}^{\mathrm{a}}$ & $\sigma$ & As deposition & $500^{\circ} \mathrm{C}$ \\
\hline $\mathrm{SiO}_{2}$ & $72[46]$ & 347 & 1.44 & $0.17[47]$ & & \\
$\mathrm{Ta}_{2} \mathrm{O}_{5}$ & $140[46]$ & 236 & 2.12 & $0.23[9]$ & & \\
$\mathrm{aSi}$ & $147[48]$ & 134 & 3.73 & 0.23 & & \\
Upper stack & 99.5 & $5 \times(347+236)=2915$ & & 0.2 & $(4.29 \pm 0.24)$ & $(3.56 \pm 0.31)$ \\
Lower stack & 92.9 & $5 \times(347+134)=2405$ & & 0.2 & $(0.44 \pm 0.06)$ & $(0.28 \pm 0.06)$ \\
Full stack & 96.3 & $2915+2405=5320$ & & 0.2 & $(2.80 \pm 0.20)$ & $(2.06 \pm 0.21)$ \\
\hline \hline
\end{tabular}

ameasured for aSi (which dominates the reflectivity) single layers produced by the same vendor via fitting of transmission spectra: $n$ changes by $<2 \%$ with heat treatment and $n \times t$ by $<1 \%$. 
due to the differing thermomechanical properties of the different coating materials. Our coating provides almost a factor of 2 less thermal noise compared to a full $\mathrm{SiO}_{2} / \mathrm{Ta}_{2} \mathrm{O}_{5}$ coating deposited using the same process, while successfully suppressing the optical absorption contribution of the $\mathrm{SiO}_{2} / \mathrm{aSi}$ layers by more than a factor of 20 .

This coating design was optimized to demonstrate the principle of multimaterial coatings and is unlikely to be suitable for use in a certain gravitational-wave detector as it is, as it was not designed and optimized for this purpose. This experimental verification is a significant step towards the realization of proposed multimaterial coating designs for the next generations of gravitational-wave detectors $[25,26,31,34]$ to enable the required thermal noise reduction.

We are grateful for financial support from STFC (ST/N005422/1), the Royal Society (RG110331), the University of Glasgow and the Deutsche Forschungsgemeinschaft (DFG, German Research Foundation, STE 2646/1-1). I. W. M. was supported by a Royal Society Research Fellowship while working on this Letter. We are grateful to the International Max Planck Partnership for Measurement and Observation at the Quantum Limit for support, and we thank our colleagues in the LSC and the Virgo Collaboration and within SUPA for their interest in this work. This Letter has LIGO Document No. LIGO-P1900002.

*Jessica.Steinlechner@ligo.org

[1] B. P. Abbott, R. Abbott, T. D. Abbott, M. R. Abernathy, F. Acernese, K. Ackley, C. Adams, T. Adams, P. Addesso, R. X. Adhikari et al. (LIGO Scientific Collaboration and Virgo Collaboration), Observation of Gravitational Waves from a Binary Black Hole Merger, Phys. Rev. Lett. 116, 061102 (2016).

[2] B. P. Abbott, R. Abbott, T. D. Abbott, M. R. Abernathy, F. Acernese, K. Ackley, C. Adams, T. Adams, P. Addesso, R. X. Adhikari et al. (LIGO Scientific Collaboration and Virgo Collaboration), GW151226: Observation of Gravitational Waves from a 22-Solar-Mass Binary Black Hole Coalescence, Phys. Rev. Lett. 116, 241103 (2016).

[3] B. P. Abbott, R. Abbott, T. D. Abbott, F. Acernese, K. Ackley, C. Adams, T. Adams, P. Addesso, R. X. Adhikari, and V. B. Adya et al. (LIGO Scientific Collaboration and Virgo Collaboration), GW170104: Observation of a 50-Solar-Mass Binary Black Hole Coalescence at Redshift 0.2, Phys. Rev. Lett. 118, 221101 (2017).

[4] B. P. Abbott, R. Abbott, T. D. Abbott, F. Acernese, K. Ackley, C. Adams, T. Adams, P. Addesso, R. X. Adhikari, and V. B. Adya et al. (LIGO Scientific Collaboration and Virgo Collaboration), GW170814: A Three-Detector Observation of Gravitational Waves from a Binary Black Hole Coalescence, Phys. Rev. Lett. 119, 141101 (2017).

[5] B. P. Abbott, R. Abbott, T. D. Abbott, M. R. Abernathy, F. Acernese, K. Ackley, C. Adams, T. Adams, P. Addesso, R. X. Adhikari et al. (LIGO Scientific Collaboration and
Virgo Collaboration), GWTC-1: A Gravitational-Wave Transient Catalog of Compact Binary Mergers Observed by LIGO and Virgo during the First and Second Observing Runs, Phys. Rev. X 9, 031040 (2019).

[6] B. P. Abbott, R. Abbott, T. D. Abbott, F. Acernese, K. Ackley, C. Adams, T. Adams, P. Addesso, R. X. Adhikari, V. B. Adya et al. (LIGO Scientific Collaboration and Virgo Collaboration), GW170817: Observation of Gravitational Waves from a Binary Neutron Star Inspiral, Phys. Rev. Lett. 119, 161101 (2017).

[7] B. P. Abbott, R. Abbott, T. D. Abbott, M. R. Abernathy, F. Acernese, K. Ackley, C. Adams, T. Adams, P. Addesso, R. X. Adhikari et al. (LIGO Scientific Collaboration and Virgo Collaboration), GW150914: The Advanced LIGO Detectors in the Era of First Discoveries, Phys. Rev. Lett. 116, 131103 (2016).

[8] F. Acernese et al., Advanced Virgo: a second-generation interferometric gravitational wave detector, Classical Quantum Gravity 32, 024001 (2015).

[9] M. Abernathy et al., Report No. ET-0106C-10, 2011.

[10] Y. Aso, Y. Michimura, K. Somiya, M. Ando, O. Miyakawa, T. Sekiguchi, D. Tatsumi, and H. Yamamoto (LIGO Scientific Collaboration and Virgo Collaboration), Interferometer design of the KAGRA gravitational wave detector, Phys. Rev. D 88, 043007 (2013).

[11] G. M. Harry, H. Armandula, E. Black, D. R. M. Crooks, G. Cagnoli, J. Hough, P. Murray, S. Reid, S. Rowan, P. Sneddon, M. M. Fejer, R. Route, and S. D. Penn, Thermal noise from optical coatings in gravitational wave detectors, Appl. Opt. 45, 1569 (2006).

[12] G. M. Harry et al., Titania-doped tantala/silica coatings for gravitational-wave detection, Classical Quantum Gravity 24, 405 (2007).

[13] R. Flaminio, J. Franc, C. Michel, N. Morgado, L. Pinard, and B. Sassolas, A study of coating mechanical and optical losses in view of reducing mirror thermal noise in gravitational wave detectors, Classical Quantum Gravity 27, 084030 (2010).

[14] M. Granata, E. Saracco, N. Morgado, A. Cajgfinger, G. Cagnoli, J. Degallaix, V. Dolique, D. Forest, J. Franc, C. Michel, L. Pinard, and R. Flaminio, Mechanical loss in state-of-the-art amorphous optical coatings, Phys. Rev. D 93, 012007 (2016).

[15] M. J. Hart, R. Bassiri, K. B. Borisenko, M. Véron, E. F. Rauch, I. W. Martin, S. Rowan, M. M. Fejer, and I. MacLaren, Medium range structural order in amorphous tantala spatially resolved with changes to atomic structure by thermal annealing, J. Non-Cryst. Solids 438, 10 (2016).

[16] J. P. Trinastic, R. Hamdan, C. Billman, and H.-P. Cheng, Molecular dynamics modeling of mechanical loss in amorphous tantala and titania-doped tantala, Phys. Rev. B 93, 014105 (2016).

[17] R. Bassiri, M. R. Abernathy, F. Liou, A. Mehta, E. K. Gustafson, M. J. Hart, H. N. Isa, N. Kim, A. C. Lin, I. MacLaren, I. W. Martin, R. K. Route, S. Rowan, B. Shyam, J. F. Stebbins, and M. M. Fejer, Order, disorder and mixing: The atomic structure of amorphous mixtures of titania and tantala, J. Non-Cryst. Solids 438, 59 (2016).

[18] M. Granata, E. Coillet, V. Martinez, V. Dolique, A. Amato, M. Canepa, J. Margueritat, C. Martinet, A. Mermet, C. Michel, L. Pinard, B. Sassolas, and G. Cagnoli, Correlated 
evolution of structure and mechanical loss of a sputtered silica film, Phys. Rev. Mater. 2, 053607 (2018).

[19] A. Amato, S. Terreni, M. Granata, C. Michel, B. Sassolas, L. Pinard, M. Canepa, and G. Cagnoli, Observation of a correlation between internal friction and urbach energy in amorphous oxides thin films, Sci. Rep. 10, 1670 (2020).

[20] G. Vajente et al., Effect of elevated substrate temperature deposition on the mechanical losses in tantala thin film coatings, Classical Quantum Gravity 35, 075001 (2018).

[21] A. Amato, G. Cagnoli, M. Canepa, E. Coillet, J. Degallaix, V. Dolique, D. Forest, M. Granata, V. Martinez, C. Michel, L. Pinard, B. Sassolas, and J. Teillon, High-reflection coatings for gravitational-wave detectors: State of the art and future developments, J. Phys. Conf. Ser. 957, 012006 (2018).

[22] H.-W. Pan, S.-J. Wang, L.-C. Kuo, S. Chao, M. Principe, I. M. Pinto, and R. DeSalvo, Thickness-dependent crystallization on thermal anneal for titania/silica nm-layer composites deposited by ion beam sputter method, Opt. Express 22, 29847 (2014).

[23] M. Magnozzi, S. Terreni, L. Anghinolfi, S. Uttiya, M. Carnasciali, G. Gemme, M. Neri, M. Principe, I. Pinto, L.-C. Kuo, S. Chao, and M. Canepa, Optical properties of amorphous $\mathrm{SiO}_{2}-\mathrm{TiO}_{2}$ multi-nanolayered coatings for 1064-nm mirror technology, Opt. Mater. 75, 94 (2018).

[24] G. D. Cole, W. Zhang, B. J. Bjork, D. Follman, P. Heu, C. Deutsch, L. Sonderhouse, J. Robinson, C. Franz, A. Alexandrovski, M. Notcutt, O. H. Heckl, J. Ye, and M. Aspelmeyer, High-performance near- and mid-infrared crystalline coatings, Optica 3, 647 (2016).

[25] H.-W. Pan, L.-C. Kuo, L.-A. Chang, S. Chao, I. W. Martin, J. Steinlechner, and M. Fletcher, Silicon nitride and silica quarter-wave stacks for low-thermal-noise mirror coatings, Phys. Rev. D 98, 102001 (2018).

[26] K. Craig, J. Steinlechner, P. G. Murray, A. S. Bell, R. Birney, K. Haughian, J. Hough, I. MacLaren, S. Penn, S. Reid, R. Robie, S. Rowan, and I. W. Martin, Mirror Coating Solution for the Cryogenic Einstein Telescope, Phys. Rev. Lett. 122, 231102 (2019).

[27] V. Pierro, V. Fiumara, F. Chiadini, F. Bobba, G. Carapella, C. D. Giorgio, O. Durante, R. Fittipaldi, E. M. Villa, J. Neilson, M. Principe, and I. Pinto, On the performance limits of coatings for gravitational wave detectors made of alternating layers of two materials, Opt. Mater. 96, 109269 (2019).

[28] X. Liu, B. E. White, Jr., R. O. Pohl, E. Iwanizcko, K. M. Jones, A. H. Mahan, B. N. Nelson, R. S. Crandall, and S. Veprek, Amorphous Solid without Low Energy Excitations, Phys. Rev. Lett. 78, 4418 (1997).

[29] J. Steinlechner, I. W. Martin, R. Bassiri, A. Bell, M. M. Fejer, J. Hough, A. Markosyan, R. K. Route, S. Rowan, and Z. Tornasi, Optical absorption of ion-beam sputtered amorphous silicon coatings, Phys. Rev. D 93, 062005 (2016).

[30] J. Steinlechner, I. W. Martin, A. S. Bell, J. Hough, M. Fletcher, P. G. Murray, R. Robie, S. Rowan, and R. Schnabel, Silicon-Based Optical Mirror Coatings for Ultrahigh Precision Metrology and Sensing, Phys. Rev. Lett. 120, 263602 (2018).

[31] R. Birney, J. Steinlechner, Z. Tornasi, S. MacFoy, D. Vine, A. S. Bell, D. Gibson, J. Hough, S. Rowan, P. Sortais, S. Sproules, S. Tait, I. W. Martin, and S. Reid, Amorphous
Silicon with Extremely Low Absorption: Beating Thermal Noise in Gravitational Astronomy, Phys. Rev. Lett. 121, 191101 (2018).

[32] J. Steinlechner, I. W. Martin, J. Hough, C. Krüger, S. Rowan, and R. Schnabel, Thermal noise reduction and absorption optimization via multimaterial coatings, Phys. Rev. D 91, 042001 (2015).

[33] W. Yam, S. Gras, and M. Evans, Multimaterial coatings with reduced thermal noise, Phys. Rev. D 91, 042002 (2015).

[34] J. Steinlechner, C. Krüger, I. W. Martin, A. Bell, J. Hough, H. Kaufer, S. Rowan, R. Schnabel, and S. Steinlechner, Optical absorption of silicon nitride membranes at $1064 \mathrm{~nm}$ and at $1550 \mathrm{~nm}$, Phys. Rev. D 96, 022007 (2017).

[35] J. Wu and C. C. Yu, How stress can reduce dissipation in glasses, Phys. Rev. B 84, 174109 (2011).

[36] http://www.tafelmaier.de/index.php? page $=$ beschichtung \&lng=en (2016).

[37] The disks were single-side coated resulting in a small, stress-induced concave curvature (towards the coating) of $R \approx-200 \mathrm{~m}$ for the partial stacks and $R \approx-100 \mathrm{~m}$ for the full stack after optimum heat treatment. For such small curvatures, previous results suggest there is unlikely to be any significant effect on the mechanical loss of the coating [14].

[38] Mechanical loss of a $\mathrm{Si}$ [30], $\mathrm{SiO}_{2}$ [39], and $\mathrm{Ta}_{2} \mathrm{O}_{5}$ [20] has also been observed to reduce with heat treatment.

[39] R. Robie, Characterisation of the mechanical properties of thin-film mirror coating materials for use in future interferometric gravitational wave detectors, Ph.D. thesis, University of Glasgow, 2018.

[40] Fewer temperatures were studied for the upper stack as we did not have enough samples on low-absorption Corning 7979 substrates, and the absorption of this stack was so low that the use of higher-absorbing Corning 7980 substrates was not feasible.

[41] A. Alexandrovski, M. Fejer, A. Markosian, and R. Route, Photothermal common-path interferometry (PCI): new developments, Proc. SPIE Int. Soc. Opt. Eng. 7193, 71930D (2009).

[42] E. Cesarini, M. Lorenzini, E. Campagna, F. Martelli, F. Piergiovanni, F. Vetrano, G. Losurdo, and G. Cagnoli, A "gentle" nodal suspension for measurements of the acoustic attenuation in materials, Rev. Sci. Instrum. 80, 053904 (2009).

[43] G. M. Harry, A. M. Gretarsson, P. R. Saulson, S. E. Kittelberger, S. D. Penn, W. J. Startin, S. Rowan, M. M. Fejer, D. R. M. Crooks, G. Cagnoli, J. Hough, and N. Nakagawa, Thermal noise in interferometric gravitational wave detectors due to dielectric optical coatings, Classical Quantum Gravity 19, 897 (2002).

[44] T. Hong, H. Yang, E. K. Gustafson, R. X. Adhikari, and Y. Chen, Brownian thermal noise in multilayer coated mirrors, Phys. Rev. D 87, 082001 (2013).

[45] D. R. M. Crooks, G. Cagnoli, M. M. Fejer, A. Gretarsson, G. Harry, J. Hough, N. Nakagawa, S. Penn, R. Route, S. Rowan, and P. H. Sneddon, Experimental measurements of coating mechanical loss factors, Classical Quantum Gravity 21, S1059 (2004).

[46] M. M. Fejer, S. Rowan, G. Cagnoli, D. R. M. Crooks, A. Gretarsson, G. M. Harry, J. Hough, S. D. Penn, P. H. 
Sneddon, and S. P. Vyatchanin, Thermoelastic dissipation in inhomogeneous media: loss measurements and displacement noise in coated test masses for interferometric gravitational wave detectors, Phys. Rev. D 70, 082003 (2004).

[47] https://www.heraeus.com/en/hca/fused_silica_quartz_ knowledge_base_1/properties_1/properti es_hca.html.

[48] M. R. Abernathy, Mechanical properties of coating materials for use in the mirrors of interferometric gravitational wave detectors, Ph.D. Thesis, University of Glasgow, 2012.
[49] M. Granata, A. Amato, L. Balzarini, M. Canepa, J. Degallaix, D. Forest, V. Dolique, L. Mereni, C. Michel, L. Pinard, B. Sassolas, J. Teillon, and G. Cagnoli, Amorphous optical coatings of present gravitational-wave interferometers, Classical Quantum Gravity 37, 095004 (2020).

[50] It should be noted that the $\mathrm{Ta}_{2} \mathrm{O}_{5}$ studied here was relatively lossy, and for the best $\mathrm{Ta}_{2} \mathrm{O}_{5}$, a smaller improvement factor would be obtained.

[51] C. Wipf, N. Demos, G. Vajente, and A. Gupta (private communication). 